\title{
Monolithically Integrated Electrically Pumped Continuous-Wave III-V Quantum Dot Light Sources on Silicon
}

\author{
Mengya Liao, Student Member, IEEE, Siming Chen, Member, IEEE, Suguo Huo, Si Chen, Jiang Wu, \\ Mingchu Tang, Member, IEEE, Ken Kennedy, Wei Li, Saurabh Kumar, Mickael Martin, Thierry Baron, \\ Chaoyuan Jin, Ian Ross, Alwyn Seeds, Fellow, IEEE, and Huiyun Liu
}

(Invited Paper)

\begin{abstract}
In this paper, we report monolithically integrated III$\mathrm{V}$ quantum dot (QD) light-emitting sources on silicon substrates for silicon photonics. We describe the first practical InAs/GaAs QD lasers monolithically grown on an offcut silicon (001) substrate due to the realization of high quality III-V epilayers on silicon with low defect density, indicating that the large material dissimilarity between III-Vs and silicon is no longer a fundamental barrier limiting monolithic growth of III-V lasers on $\mathrm{Si}$ substrates. Although the use of offcut silicon substrates overcomes the antiphase boundary (APB) problem, it has the disadvantage of not being readily compatible with standard microelectronics fabrication, where wafers with on-axis silicon (001) substrates are used. We therefore report, to the best of our knowledge, the first electrically pumped continuous-wave (c.w.) InAs/GaAs QD lasers fabricated on on-axis $\mathrm{GaAs} / \mathrm{Si}(001)$ substrates without any intermediate buffer layers. Based on the achievements described above, we move on to report the first study of post-fabrication and prototyping of various Si-based light emitting sources by utilizing the focused ion beam (FIB) technique, with the intention of expediting the progress toward large-scale and low-cost photonic integrated circuits monolithically integrated on a silicon platform. We compare two Si-based QD lasers with as-cleaved and FIB-made facets, and prove that FIB is a powerful tool to fabricate integrated
\end{abstract}

Manuscript received January 30, 2017; revised March 24, 2017; accepted April 5, 2017. This work is supported by UK EPSRC under Grant EP/J012904/1, Grant EP/K029118/1, and Grant EP/P006973/1. The work of M. Liao was supported by China Scholarship Council. (Corresponding author: Huiyun Liu.)

M. Liao, S-M. Chen, J. Wu, M. Tang, A. Seeds, and H. Liu are with the Department of Electronic and Electrical Engineering, University College London, London WC1E 7JE, U.K. (e-mail: mengya.liao@ucl.ac.uk; siming.chen@ucl.ac.uk; jiang.wu@ucl.ac.uk; mingchu.tang.11@ucl.ac.uk; a.seeds@ucl.ac.uk; huiyun.liu@ucl.ac.uk).

S. Huo is with London Centre for Nanotechnology, University College London, London WC1H 0AH, U.K. (e-mail: s.huo@ucl.ac.uk).

S. Chen, W. Li, C. Jin, and I. Ross are with the Department of Electronic and Electrical Engineering, University of Sheffield, Sheffield S3 7JD, U.K. (e-mail: schen62@sheffield.ac.uk; wli9@sheffield.ac.uk; c.jin@sheffield.ac.uk; i.ross@sheffield.ac.uk).

K. Kennedy and S. Kumar are with the EPSRC National Centre for III-V Technologies, Department of Electronic and Electrical Engineering, University of Sheffield, Sheffield S3 7HQ, U.K. (e-mail: k.kennedy@ sheffield.ac.uk; saurabh.kumar@sheffield.ac.uk).

M. Martin and T. Baron are with the Universite Grenoble Alpes, CNRS, CEA-LETI, MINATEC Campus, Grenoble F-38054, France (e-mail: mickael.martin@cea.fr; thierry.baron@cea.fr).

Color versions of one or more of the figures in this paper are available online at http://ieeexplore.ieee.org.

Digital Object Identifier 10.1109/JSTQE.2017.2693025 lasers on silicon substrates. Using angled facet structures, which effectively reduce facet reflectivity, we demonstrate Si-based InAs/GaAs QD superluminescent light emitting diodes (SLDs) operating under c.w. conditions at room temperature for the first time. The work described represents significant advances towards the realization of a comprehensive silicon photonics technology.

Index Terms-Molecular beam epitaxy, quantum dots, semiconductor lasers, silicon photonics, focused ion beam.

\section{INTRODUCTION}

$\mathbf{O}$ VER the last few years, an almost exponential growth in global internet traffic has raised significant challenges for the modern Datacentre and Datacom industries, which require an efficient interconnection framework to support the large bandwidth demand with low power consumption. These demanding requirements have created a major "bottleneck" for conventional copper interconnections in transporting digital information on different scales, ranging from worldwide links to inter- and intra-chip communications [1], [2]. The integration of optical interconnects with photonic integrated circuits (PICs) on a silicon platform has become one of the most promising candidates to solve this problem [3]-[10]. There have been prototype demonstrations to achieve large bandwidth through integrating wavelength division multiplexing (WDM) systems into the PICs [11], [12]. This has traditionally been done using an array of laser diodes (LDs) emitting at different wavelengths which are then combined by an optical multiplexer into one output fiber. Based on this technique, the first Intel Silicon Photonics $100 \mathrm{G}$ optical transceiver products have now been shipped in volume, targeting the need for faster connections from rack to rack and across Datacentre [13]. In addition to lasers, superluminescent light-emitting diodes (SLDs), an edge emitting semiconductor light source, that combines the high output power with broad optical spectrum bandwidth, have attracted tremendous attention for a wide range of applications [14], [15], especially in WDM systems [16]. The use of SLDs in a high-density WDM system allows all subscribers to utilize an identical transmitter through use of spectrum-slicing techniques [17], [18], where broad emission spectrum from a single SLD can be 'sliced' into 
many different wavelengths to satisfy all the required channels, therefore the number of active light sources on PICs can be significantly reduced leading to lower power consumption and heat dissipation. This is one of the major advantages of SLDs over LDs and other competitors for use in WDM systems to meet future Tera-bit links.

To fully exploit the economies of scale of silicon manufacturing environments, and to achieve low-cost, massive scalable integration, development of CMOS compatible Si-based light sources is of considerable importance [19]-[21]. However, Si, like $\mathrm{Ge}$, is an indirect bandgap material, and is therefore an inefficient light emitter [2], [3]. Integration of III-V materials with $\mathrm{Si}$ substrates, provides the best alternative to solve the lasing issue on Si substrates. To date, wafer bonding has achieved a dominance in Si photonic integration area [3], [22], [23], as it allows for the combination of light emitting capability of III-V materials with light routing of $\mathrm{Si}$ waveguide. Notably, smaller cavity length lasers have successfully been realized by using integrated mirrors, such as distributed Bragg reflectors [24], [25], reflective multimode interferometers [26] or ring structure, which allows for the formation of large number of channels, while, with reduced crosstalk between adjacent channels as well as laser cavity without the requirement for polishing facets [23]. In addition to wafer bonding, monolithic growth method is another rapid-growing research area due to its cost-effective and massive scalable integration merits. [27]. However, the large material dissimilarity between III-Vs and group IV materials is a severe obstacle facing monolithic growth, including lattice mismatch, thermal expansion coefficient differences, and polar versus nonpolar surfaces. These differences between III-V and Group IV materials tend to produce various types of defectsAPBs, threading dislocations (TDs), and microcracks - which all generate nonradiative recombination centers and dramatically undermine the promise of III-V materials [28]. Over the last two decades, III-V QD light sources, including LDs and SLDs, have been demonstrated with much lower threshold current density [29]-[31] and significantly reduced temperature sensitivity [32] than III-V quantum well (QW) devices due to their delta-function-like density of states [33], [34]. Also, the superiority of QD structures for achieving broad bandwidth in SLDs is well established owing to their naturally large size inhomogeneity when grown by the S-K growth mode [35]. More recently, the use of QDs as the active region has shown their advantages for monolithic growth of III-V light sources on Ge [8], [36], Ge-on-Si [9], [37], [38], and Si [39]-[43] substrates due to their reduced sensitivity to defects [44], [45]. In particular, preliminary yield and reliability data on the Ge-on-Si based QD lasers have been reported, achieving an extrapolated lifetime of 4,627 hours [46]. However, such an intermediate Ge layer limits the efficient light coupling from this layer to a silicon waveguide due to the large optical absorption coefficient of $\mathrm{Ge}$ at telecommunications wavelengths [47]. To bypass the need for the absorptive Ge intermediate buffer layer, in our previous work, by optimizing the initial growth temperature of GaAs nucleation layer (NL) as well as inserting InGaAs/GaAs strained layer superlattices (SLSs) serving as dislocation filter layers (DFLs), we have realized the first electrically pumped
$1.3 \mu \mathrm{m}$ InAs/GaAs QD lasers grown on silicon substrates [39]. Following on from this work, the performance of Si-based QD lasers has been gradually improved due to the efficient reduction in the defect density [43], [48], [49]. However, a high performance long lifetime electrically pumped c.w. III-V QD laser monolithically grown on silicon substrate has only recently been demonstrated [38].

In our previous work, $\mathrm{Si}(001)$ wafers with an offcut of $4^{\circ}$ to the [011] plane have been used to prevent the formation of antiphase domains (APDs) while growing polar III-V materials on non-polar group-IV substrates. Although the offcut could be successfully used for annihilation of APDs, it compromises full compatibility with standard microelectronics fabrication, where on-axis Si (001) substrates are typically used [50], [51]. Recently, there has been much progress for InAs QD lasers on Si (001) substrates, including the demonstrations of optically pumped microcavity lasers on patterned Si (001) substrates and electrically pumped lasers by growth on a GaP/Si (001) template [52].

In addition to Si-based lasers, we have also demonstrated the first electrically pumped InAs/GaAs QD SLDs monolithically grown on Si substrates [53]. These devices are limited to pulsed operating conditions and the two-section structure requires complex fabrication procedure. Reliable and effective SLDs on Si substrates with c.w. operation is required.

Optical lithography using photoresist is the dominant patterning technique as it allows the patterning of an entire $300 \mathrm{~mm}$ (and larger) wafer in a short time with high yield. However, due to the need for expensive masks and repeated processing, development of new devices is costly and time-consuming. It is therefore attractive to use prototyping technologies that enable rapid and flexible fabrication of nanophotonic components, ranging from micro- to nanometer scales [54]. Among these approaches, FIB is an interesting alternative as it allows photoresist-free and direct writing, which enables the post-fabrication of devices with a more complex topography such as ridge waveguides and laser facets. In addition, FIB technology has been widely used for photonics applications, including fabrication of grating couplers and various waveguides [54], [55].

In this paper, we present high-performance monolithically integrated electrically pumped c.w. InAs/GaAs QD light-emitting sources on silicon substrates. The paper is organized as follows: In Section II, we describe high performance electrically pumped c.w. operating InAs QD lasers epitaxially grown on a silicon substrate with an ultra-low threshold current density of $62.5 \mathrm{~A} / \mathrm{cm}^{2}$, single facet output power of $52.5 \mathrm{~mW}$ and over 100,000 hours lifetime [47]. In Section III, we present, to the best of our knowledge, the first electrically pumped c.w InAs/GaAs QD lasers directly grown on on-axis GaAs / Si (001) substrates (without any intermediate buffer layers) with lasing operation to over $100{ }^{\circ} \mathrm{C}$ and a single facet output power exceeding $130 \mathrm{~mW}$ at room temperature [56]. In Section IV, we report on the first study of post-processing of Si-based III-V QD lasers by means of FIB milling. We include comparison of FIB-made facets with as-cleaved facets for Si-based III-V QD lasers and show that there is no obvious deterioration between these two facet realisation approaches. Thus, Si-based light sources with 


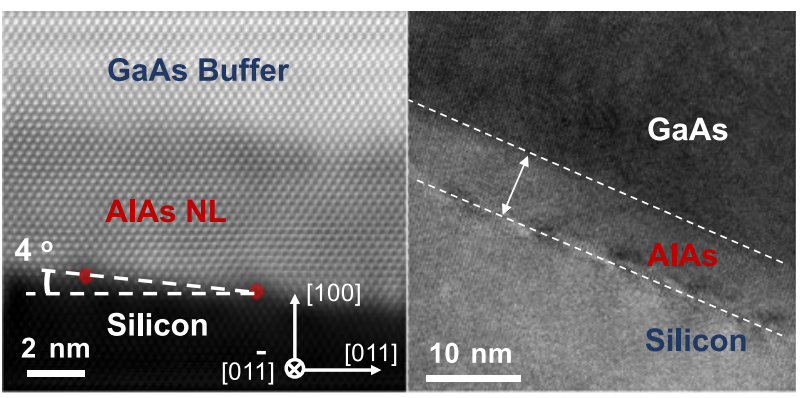

(a)

(b)

Fig. 1. (a) High-resolution scanning TEM image of the AlAs/Si interface, showing the $4^{\circ}$ offcut of silicon substrate. (b) High resolution TEM image showing the AlAs nucleation layer on $\mathrm{Si}$.

different properties can be realized by simply fabricating angled front facets with different cutting angles by FIB.

\section{Continuous Wave Quantum Dot Lasers ON Si SUBSTRATES WiTH LONG LIFETIMES}

\section{A. Material and Methods}

InAs/GaAs QD laser structures were directly grown on phosphorus-doped $\mathrm{Si}$ substrates by a solid-source molecular beam epitaxy (MBE) system. The (001)-silicon wafer with $4^{\circ}$ miscut-angle oriented towards the [011] plane was used to suppress APBs due to the formation of double-layer steps [49], as shown in Fig. 1(a). Before the initial epitaxy growth, oxide desorption was performed by thermally treating the silicon substrate at $900{ }^{\circ} \mathrm{C}$ for 30 mins, followed by depositing III-V epilayers that consists of a $6 \mathrm{~nm}$ AlAs nucleation layer (Fig. 1(b)) grown by migration-enhanced epitaxy (MEE), $1 \mu \mathrm{m}$ GaAs buffer formed by a three-step temperature growth technique and InGaAs/GaAs SLSs. The III-V epilayer employed here was previously optimized and plays a critical role in blocking the TDs [40]. To further improve the annihilation efficacy of TDs, in situ thermal annealing of the SLS was performed using the method detailed in [40] and [49]. As a result, high quality IIIV/Si epilayers with a low density of TDs on the order of $10^{5}-10^{6}$ $\mathrm{cm}^{-2}$ has been obtained [40], [57], [58]. Based on this template, a standard p-i-n laser structure was deposited on the optimized III-V/Si epilayers in the following order: a $1.4 \mu \mathrm{m}$ n-doped AlGaAs cladding layer, a $140 \mathrm{~nm}$ lower undoped AlGaAs guiding layer, a five-layer InAs/InGaAs/GaAs dots-in-well (DWELL) active region [59], a $140 \mathrm{~nm}$ undoped upper AlGaAs guiding layer, a $1.4 \mu \mathrm{m}$ p-doped AlGaAs cladding layer, and finally a $300 \mathrm{~nm}$ highly p-doped GaAs contact layer. High resolution bright-field scanning TEM measurement was employed to characterize the QD active region in [47]. A nearly defect-free active region is observed, indicating that most of the TDs generated at the III/Si interface have been effectively confined/annihilated within the III-V epilayers thanks to the strategies employed during the growth. From a typical atomic force microscopy (AFM) image for uncapped InAs QDs, grown on the III-V epilayers, a dot density of $\sim 3.0 \times 10^{10} \mathrm{~cm}^{-1}$ with good uniformity was obtained, consequently a strong room temperature photoluminescence (PL) emission at $\sim 1300$ nm with a narrow linewidth of $\sim 29 \mathrm{meV}$ has been achieved in [47].

Broad-area lasers were fabricated following the standard lithography, wet etching and metallization techniques as described in [47]. The fabricated laser bars were then mounted on copper heat-sinks and gold-wire-bonded to enable testing.

\section{B. Result and Discussion}

Fig. 2(a) shows the light-current (LI) characteristics of a typical InAs/GaAs QD laser on a Si substrate under c.w. operation at room temperature. An extremely low threshold current density of $62.5 \mathrm{~A} / \mathrm{cm}^{2}$ and a high output power of over $52.5 \mathrm{~mW}$ has been achieved from a single facet The c.w. lasing spectrum measured at an injection current density of $225 \mathrm{~A} / \mathrm{cm}^{2}$ is shown in the inset of Fig. 2(a), where the lasing peak at $1316 \mathrm{~nm}$ is observed. Moreover, the maximum lasing temperature of the InAs/GaAs QD laser is up to $75^{\circ} \mathrm{C}$ under c.w. mode and $120^{\circ} \mathrm{C}$ under pulsed operation. The extrapolated characteristic temperature $\left(\mathrm{T}_{0}\right)$ is $\sim 51 \mathrm{~K}$ between 20 and $60{ }^{\circ} \mathrm{C}$ and $\sim 35 \mathrm{~K}$ between 70 and $120^{\circ} \mathrm{C}$.

Fig. 2(b) shows the ageing data for this InAs/GaAs QD laser on silicon. The device was aged in auto current control mode at $26{ }^{\circ} \mathrm{C}$ under $210 \mathrm{~mA}$ of constant applied current. A $27.5 \%$ increase in threshold over $3100 \mathrm{~h}$ is observed with $14.4 \%$ increase occurring in the first $500 \mathrm{~h}$, followed by a very slow increment of threshold over time. A sub-linear mode model is employed to fit the aging data [46] (where we define the mean time to failure as a $100 \%$ increased threshold) and an extrapolated lifetime of over $100,158 \mathrm{~h}$ was determined.

\section{QUANTUM Dot LASERS DIRECTLY GROWN ON EXACT (001) SILICON SUBSTRATES}

\section{A. Material and Methods}

In this work, both metal-organic chemical vapor deposition (MOCVD) and MBE machines have been used to complete the QD laser structure on silicon (001) substrates. As shown in Fig. 3(a), a thin GaAs nucleation layer is first grown directly on the $300 \mathrm{~mm}$ on-axis $\mathrm{Si}$ (001) substrates, followed by GaAs buffer layer, both layers being grown by MOCVD [50]. The full InAs/GaAs QD laser structure was then grown on the optimal GaAs/silicon (001) substrates by MBE in the following order: a $600 \mathrm{~nm}$ GaAs buffer layer, InGaAs/GaAs dislocation filter layers, and five layers of InAs/GaAs dot-in-a-well (DWELL) structures separated by $50 \mathrm{~nm}$ GaAs spacers in the middle of $30 \mathrm{~nm}$ undoped GaAs guiding layers between $1.4 \mu \mathrm{m}$ n-type lower and p-type upper $\mathrm{Al}_{0.4} \mathrm{Ga}_{0.6}$ As cladding layers. Finally, a $300 \mathrm{~nm}$ p-type GaAs contact layer was grown [56].

The structural properties of the GaAs film layer grown on Si (001) by MOCVD and InAs QDs grown on GaAs/Si (001) template by MBE are characterized by AFM measurements as shown in Fig. 3(b) and (c). Fig. 3(b) shows a typical $5 \times 5 \mu \mathrm{m}^{2}$ AFM image of $400 \mathrm{~nm}$ GaAs film layer directly grown on on-axis Si (001) substrates by MOCVD. As seen a small rootmean-square (RMS) surface roughness of $0.86 \mathrm{~nm}$ is obtained. This small roughness together with the absence of obvious 




(a)

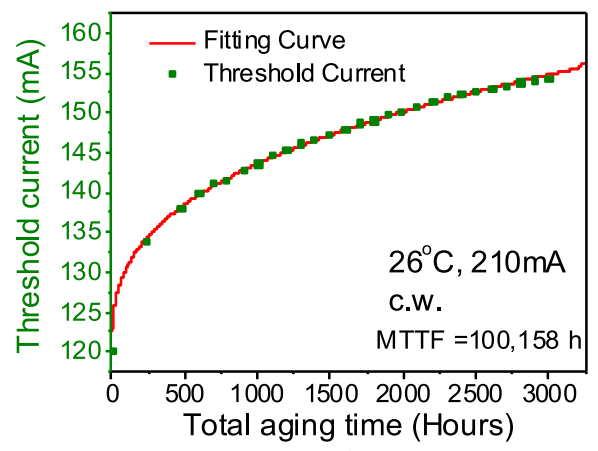

(b)

Fig. 2. (a) LIV characteristics for a Si-based InAs/GaAs QD devices under c.w. operation at room temperature. The inset shows the emission spectrum of Si-based InAs/GaAs QD laser under c.w. mode with $225 \mathrm{~A} / \mathrm{cm}^{2}$ injection current density at room temperature. (b) Lifetime test of Si-based QD laser at a c.w. drive current of $210 \mathrm{~mA}$. The red line is the best fitting line of threshold current.

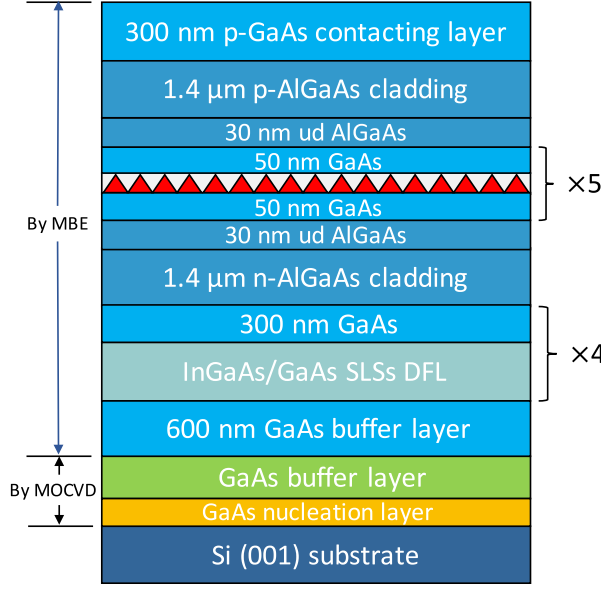

(a)



$-10 \mathrm{~nm}$



(e)

Fig. 3. (a) Schematic of the layer structure grown on $\mathrm{Si}$ (001) substrates, wherein $400 \mathrm{~nm}$ APB-free GaAs was grown by MOCVD. A typical 5-layer QD laser structure was then grown on the GaAs-on-Si virtual substrate in a MBE system. (b) A typical $5 \times 5 \mu \mathrm{m}^{2}$ AFM image of $400 \mathrm{~nm}$ GaAs grown on Si (001) substrate by MOCVD. (c) $1 \times 1 \mu \mathrm{m}^{2} \mathrm{AFM}$ image of InAs/GaAs quantum dots grown on (001) Si substrate. (d) TEM cross section image of five layers of quantum dots. (e) PL of InAs/GaAs QD lasers grown on $\mathrm{Si}(001)$ substrate.

"V"-groove features indicate that an APD-free GaAs film layer has been achieved on $\mathrm{Si}(001)$ substrates by MOCVD [50]. The typical AFM image of uncapped InAs QDs grown on GaAs/silicon (001) substrates by MBE is shown in Fig. $3(\mathrm{c})$, where a typical dot density of $3 \times 10^{10} \mathrm{~cm}^{-2}$ is measured by AFM and a good dot uniformity has also been obtained. The cross-sectional TEM image of DWELL active region is presented in Fig. 3(d), showing a nearly defect-free active region. The PL of the InAs/GaAs QD laser structure grown on GaAs/silicon (001) substrates is shown in Fig. 3(e) where the $\mathrm{PL}$ emission peak at $\sim 1285 \mathrm{~nm}$ with a linewidth of $32 \mathrm{meV}$ is observed.

\section{B. Result and Discussion}

Fig. 4(a) shows the LIV measurements for an InAs/GaAs QD laser grown on a silicon (001) substrate under c.w. operation at room temperature. A clear knee behavior in the LI curve is observed at the lasing threshold of $425 \mathrm{~A} / \mathrm{cm}^{2}$. The measured single facet output power is $43 \mathrm{~mW}$ at an injection current density of

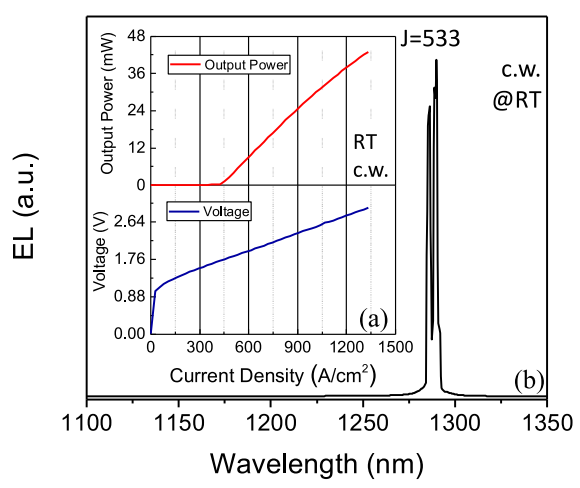

Fig. 4. (a) LIV characteristics for InAs/GaAs QD laser on exact Si substrate measured under c.w. operation at room temperature. (b) Emission spectrum of QD laser when the injection current density is $533 \mathrm{~A} / \mathrm{cm}^{2}$.

$1.3 \mathrm{kA} / \mathrm{cm}^{2}$, with no obvious evidence of power saturation up to this current density. The lasing spectrum measured at an injection current density of $533 \mathrm{~A} / \mathrm{cm}^{2}$ is shown in Fig. 4(b), in which a lasing peak at $1288 \mathrm{~nm}$ is observed. 


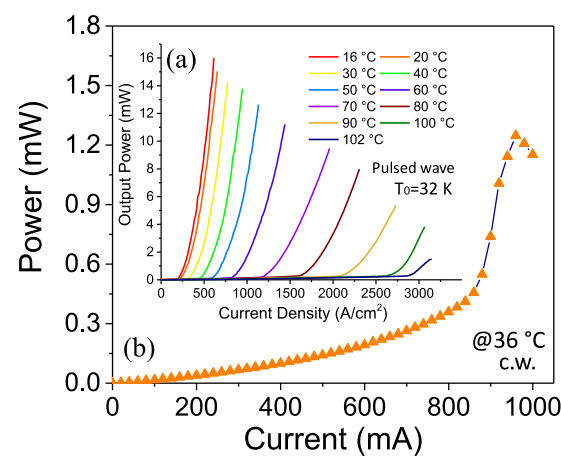

Fig. 5. LI characteristics for Si-based InAs/GaAs QD laser grown on exact $\mathrm{Si}$ substrate under pulsed wave operation with different temperature. (b) LI curve of QD laser at a heat sink temperature of $36^{\circ} \mathrm{C}$ under c.w. operation.

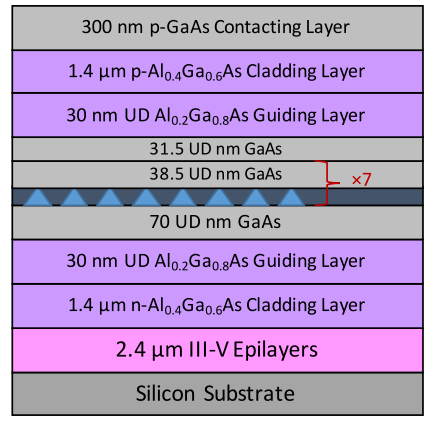

(a)

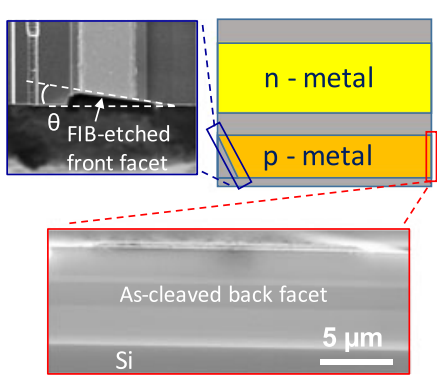

(b)
Fig. 6. (a) Schematic diagram of InAs QD laser structure grown on Si substrates. (b) Top view of the schematic diagram of the fabricated laser structure. The top-left SEM image shows the typical FIB-made (top-view) front facet of Si-based InAs QD laser. The bottom-right cross-sectional SEM image shows the typical as-cleaved back facet of the Si-based InAs QD laser.

Fig. 5(a) shows the output power against the current density for the QD laser grown on Si (001) at various heat-sink temperatures under pulsed operation. The lasing operation can be maintained up to $102{ }^{\circ} \mathrm{C}$ and the calculated $\mathrm{T}_{0}$ is $32 \mathrm{~K}$ for temperatures from $16{ }^{\circ} \mathrm{C}$ to $102^{\circ} \mathrm{C}$. This poor $\mathrm{T}_{0}$ could be improved by using modulation p-doping of the QD active region or/and a high thermal-conductivity heatsink. This silicon-based QD laser has also been tested under c.w. operation, as seen in Fig. 5(b). The maximum c.w. lasing temperature is limited to $36^{\circ} \mathrm{C}$ due to the self-heating of the device.

\section{POSTFABRicATION OF SUPERLUMINESCENT Light-EMitTing Diodes ON Si SUBSTRATES By FIB}

\section{A. Material and Methods}

In this work, the InAs/GaAs QD laser structure (as shown in Fig. 6(a)) is nominally identical to that of laser structure described in Section II except for the growth of active region. Here, in order to improve the modal gain, 7 DWELL layers have been used. After completing the growth of the laser structure, broad-area lasers of $25 \mu \mathrm{m}$ in width and $3 \mathrm{~mm}$ in length were fabricated and then mounted on copper heat-sinks and goldwire-bonded to enable testing. After device characterization as a

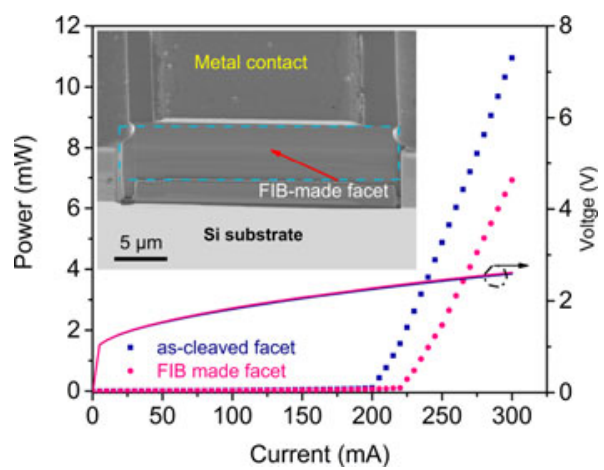

Fig. 7. LIV characteristics for Si-based InAs/GaAs QD laser with FIB-made facet and as-cleaved facets measured under c.w. operation at room temperature. The inset shows the typical SEM image of FIB-made front facet of Si-based InAs QD laser.

laser with as-cleaved facets was completed, the front as-cleaved facet was then milled, (with the back as-cleaved facet remains unchanged), by focused $\mathrm{Ga}$ ion beam [60] to form front angled facet with angles $0^{\circ}, 5^{\circ}, 8^{\circ}, 10^{\circ}, 13^{\circ}$, and $16^{\circ}$, respectively as illustrated by Fig. 6(b). The FIB milling was performed using a Ziess XB 1540 "cross beam" FIB microscope with a probe current of $500 \mathrm{pA}$ for coarse milling and $100 \mathrm{pA}$ for surface polishing. Unless stated otherwise, device characterization was performed under c.w. operation at room temperature (RT).

\section{B. Results and Discussion}

Fig. 7 compares the light-current-voltage (LIV) characteristics for a Si-based InAs/GaAs QD laser with FIB-made facet and a conventional device with as-cleaved facets under RT c.w. operation. The measured series resistances extrapolated from I-V curves were very similar between the two laser devices. The measured threshold current and slope efficiency are 200 $\mathrm{mA}$ and $0.125 \mathrm{~W} / \mathrm{A}$, respectively for the as-cleaved Si-based laser, and $222 \mathrm{~mA}$ and 0.095 W/A for the Si-based laser with FIB-made facet. Compared with as-cleaved facets device, there is no only a slight reduction in device performance for Si-based laser with FIB-made facet. The slightly degraded device performance in threshold and slope efficiency could be associated with the larger mirror loss due to ion implantation, bombardment and re-deposition arising from the FIB process when the energetic $\mathrm{Ga}^{+}$ion beam interacts with III-V sidewalls. It should be mentioned that the $\mathrm{Ga}^{+}$ion beam employed here is not the ideal ion beam source for etching GaAs/AlGaAs materials, and measurements were therefore performed as a feasibility study, not for benchmarking performance. If a proper gas-assisted FIB milling [54] or $\mathrm{He}^{+}$ion beam technique was to be used, an even better device performance would be expected. But still, as seen from the SEM image (inset of Fig. 7) of FIB etched III/V region, a clean, smooth and vertical sidewall has been obtained.

Fig. 8(a) shows the RT c.w. L-I characteristics for Si-based InAs/GaAs QD devices with different front facet angles of $0^{\circ}$, $5^{\circ}, 8^{\circ}, 10^{\circ}, 13^{\circ}$, and $16^{\circ}$, respectively. The threshold current of the FIB fabricated Si-based laser with a $0^{\circ}$ front facet angle is $222 \mathrm{~mA}$. By increasing the etched angle from $0^{\circ}$ to $5^{\circ}$, 


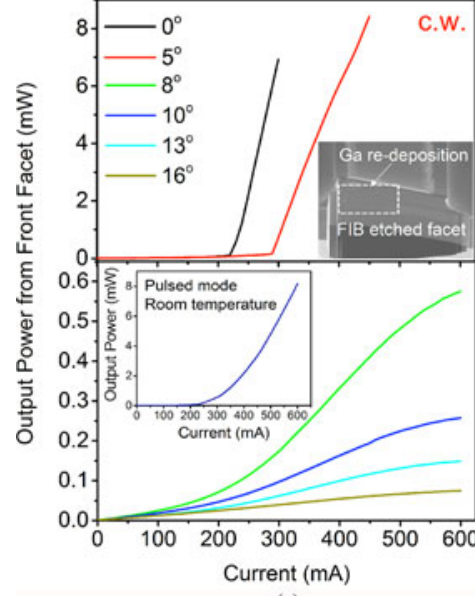

(a)

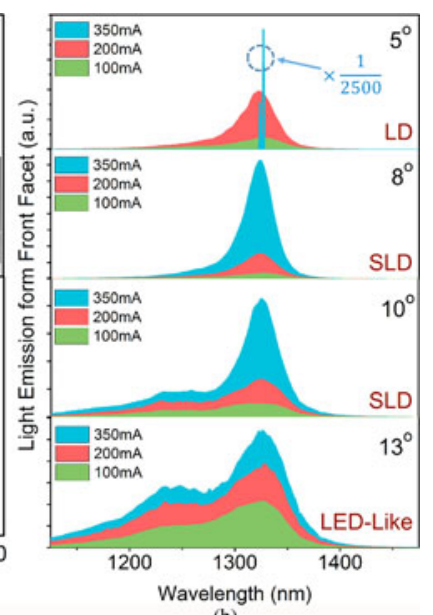

Fig. 8. Si-based device characterization. (a) L-I characteristics for a $25 \mu \mathrm{m}$ $\times 3000 \mu \mathrm{m}$ Si-based InAs/GaAs QD devices with different front facet angles of $0^{\circ}, 5^{\circ}, 8^{\circ}, 10^{\circ}, 13^{\circ}$, and $16^{\circ}$, under c.w. operation at room temperature. The inset (left) shows the L-I characteristics for this Si-based InAs/GaAs SLD laser with $8^{\circ}$ FIB-made angled facet under pulsed operation (1\% duty-cycle and $1 \mu \mathrm{s}$ pulse width) at room temperature. The inset (right) shows a cross-sectional SEM image of Si-based InAs/GaAs QD laser with $8^{\circ}$ angled front facet. (b) Room temperature EL spectra for Si-based QD devices with different front facet angles of $5^{\circ}, 8^{\circ}, 10^{\circ}$, and $13^{\circ}$ at various c.w. injection currents.

the threshold current is increased to $280 \mathrm{~mA}$, and this is due to the effectively reduced reflectivity from the angled facet, where the light beam being coupled back into guided modes has been reduced. But still the device exhibited a typical lasing characteristics. Increasing etched facet angle to $8^{\circ}$, a typical superluminescent behavior evidenced by the superlinear increase in output power with increasing current is observed. The maximum RT c.w. output power measured from the front angled facet is $0.56 \mathrm{~mW}$ at $600 \mathrm{~mA}$, where a power saturation due to over-heating of the device is observed. This Si-based QD SLD has also been examined under pulsed operation as seen in the inset (left-side) of Fig. 8(a), with limited self-heating, an output power of over $8 \mathrm{~mW}$ has been obtained at $600 \mathrm{~mA}$ where no power saturation observed up to this current injection. By stacking more layers of dots with higher dot density in each layer to increase the optical gain and also by utilizing p-type modulation doping of the QDs [61], [62], high output power with temperature-insensitive operation could be realized. In addition, the Ga ion re-deposition effect occurs during the FIB process as seen in the inset (right-side) of Fig. 8(a), which induces additional mirror loss and needs to be resolved in future devices. With further increase in the etched facet angle to $13^{\circ}$, it is noted that a nominal LED-like L-I characteristics is observed, despite the fact that the emission spectrum is still dominated by the amplified spontaneous emission (ASE), because the modal gain should have been pumped to the same level among devices with different facet angles.

Fig. 8(b) shows RT electroluminescence (EL) spectra for $\mathrm{Si}$ based QD devices with different front facet angles of $5^{\circ}, 8^{\circ}, 10^{\circ}$, and $13^{\circ}$ at various c.w. injection currents. The measured FWHM for devices with $5^{\circ}$ and $8^{\circ}$ facet angle as a function of injection current are summarized in the Fig. 9. As seen for the device with $5^{\circ}$ facet angle, at a low injection of $100 \mathrm{~mA}$, a broad spon-

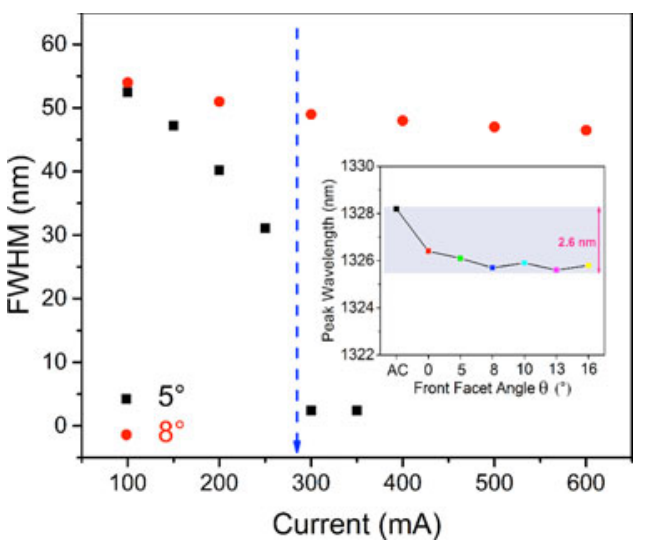

Fig. 9. The measured full-width-at-half-maximum (FWHM) for devices with $5^{\circ}$ and $8^{\circ}$ facet angles as a function of injection current. In t-inset shows the evolution of the peak wavelength (measured at $300 \mathrm{~mA}$ ) for Si-based QD devices versus the etched facet angle.

taneous emission from the ground state of QDs with a FWHM of $52.5 \mathrm{~nm}$ is obtained, as the current increases to $300 \mathrm{~mA}$, the emission peak increases suddenly in intensity and the FWHM narrows sharply to only $\sim 2.4 \mathrm{~nm}$, which indicates that lasing oscillation can still be achieved from this device, despite the effectively reduced facet reflectivity from the $5^{\circ}$ angled front facet. In contrast, for a device with $8^{\circ}$ facet angle, the measured FWHM narrows slightly with increasing injection up to $600 \mathrm{~mA}$, which suggests that the lasing has been fully inhibited in this device, and the observed spectrum narrowing effect is because: when the device working as a SLD, the modal gain is larger than the internal loss within a limited wavelength range in the middle of the gain spectrum. The spectrum is therefore dominated by the ASE within this wavelength range. Towards the edge of the gain spectrum, the wavelength-dependent gain decreases and those parts of the spectrum are dominated by spontaneous emission. The spectrum narrowing is an indirect indication of the existence of ASE. Similar amplified spontaneous emission has also been observed from the device with $10^{\circ}$ facet angle. It should be mentioned that, the $3 \mathrm{~dB}$ bandwidths reported here are relatively narrow compared to conventional InAs QD SLDs on native substrates, this is because the QD active region studied in this work is designed for lasers, where the growth conditions were intentionally optimized for small dot inhomogeneity. Improvement in bandwidth could be achieved by chirped QDs [63], QD intermixing [64], or hybrid QW/QD structures [65]. As seen from the electroluminescence (EL) spectrum for device with $13^{\circ}$ facet angle, a linear increase in spontaneous emission peak intensity with increasing current is observed, representing a nominal LED behavior (although the spectrum is still governed by ASE) due to the completely suppressed optical feedback within the FP resonator. The evolution of the peak wavelength (measured at $300 \mathrm{~mA}$ ) for Si-based QD devices versus the etched facet angle is summarized in the inset of Fig. 9. It is clear to see that the peak wavelength for Si-based QD devices with different front facet angles was almost unchanged with a value of $\sim 1326 \mathrm{~nm}$, despite the fact that device characteristics with different facet angles have been significantly modified, which indicates that FIB technology is a promising tool with large flexibility to di- 
rectly post-fabricate active photonic devices while maintaining desired communication wavelengths.

We developed a simple rate equation model to gain further insight into the suppression of the laser oscillation using angled facets. The carrier and photon dynamics is described by the following equations

$$
\begin{aligned}
\frac{d N}{d t} & =\eta_{i} \frac{I}{q V}-\left(A N+B N^{2}+C N^{3}\right)-v_{g} \Gamma g N_{p} \\
\frac{d N_{p}}{d t} & =v_{g} \Gamma g N_{p}+\beta B N^{2}-v_{g}\left(\alpha_{i}+\alpha_{m}^{\prime}\right) N_{p}
\end{aligned}
$$

where $N$ and $N_{\mathrm{p}}$ are the carrier and photon densities respectively, $A$ is the defect recombination coefficient, $B$ is the spontaneous emission coefficient, $C$ is the Auger recombination coefficient, $\Gamma$ is the optical confinement factor, $v_{g}$ is group velocity of light, $V$ is the volume of the active region, $\eta_{\mathrm{i}}$ is the internal efficiency, $\alpha_{\mathrm{i}}$ is the internal optical loss, and $\beta$ is the spontaneous emission factor. Here we adopt two different definitions of the mirror loss:

$$
\begin{aligned}
\alpha_{m} & =\frac{1}{2 L} \ln \left(\frac{1}{R_{1} R_{2}}\right) \\
\alpha_{m}^{\prime} & =\frac{1}{2 L} \ln \left(\frac{1}{R_{1} R_{2}^{\prime}}\right)
\end{aligned}
$$

the latter is called the effective mirror loss, where $L$ is the device length, $R_{1}$ is the reflectivity of the back facet, $R_{2}$ is the reflectivity of the front (angled) facet, and $R_{2}^{\prime}$ is the effective reflectivity of the front facet taking into account the coupling factor between the reflected light from the front facet and guided modes of the ridge waveguide. We assume the material gain linearly depends on the carrier density by ignoring the excessively complicated gain saturation process in SLEDs. By self-consistent iteration method the rate equations can be solved numerically, yielding an output power from the front facet

$$
P_{0}=v_{g} \alpha_{m}^{\prime} h v V_{p} N_{p} \cdot \frac{\frac{1}{\sqrt{R_{2}}}-\sqrt{R_{2}}}{\frac{1}{\sqrt{R_{2}}}-\sqrt{R_{2}}+\frac{1}{\sqrt{R_{1}}}-\sqrt{R_{1}}}
$$

Fig. 10 shows the comparison between measured L-I characteristics and simulated results by rate equations. The agreement between measurement data and simulated results is reasonably good for all facet angles at low injection levels. The obvious mismatch between the simulation and experimental results above $300 \mathrm{~mA}$ are due to the complicated thermal effects in SLEDs which lead to severer degradation of the material gain at high injection. The angle dependence of $R_{2}$ is calculated using Fresnel equations based on a simple assumption of ray optics, as shown by the curve in Fig. 11. During the modelling, we have only optimized the value of $R_{2}^{\prime}$ to obtain the best fitting results. Fig. 11 plots $R_{2}^{\prime}$ as a function of the facet angle, in which each of the $R_{2}^{\prime}$ values correspond to one of the fitting curves in Fig. 10. The increased difference between $R_{2}$ and $R_{2}^{\prime}$ suggests the coupling factor to guided modes is significant reduced with increasing facet angles, which contributes to the suppression of lasing.

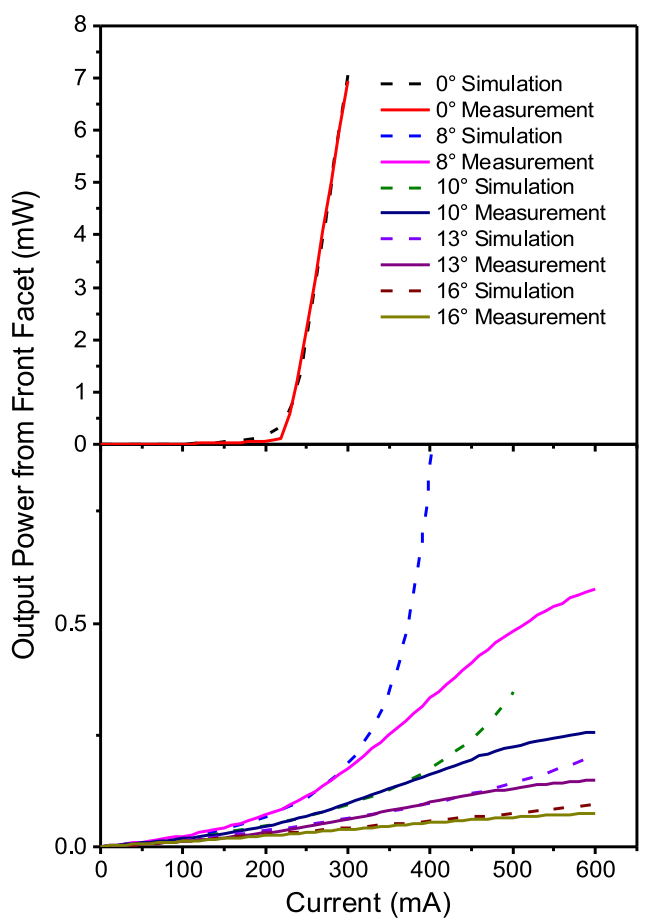

Fig. 10. The comparison between measured L-I characteristics and simulation results by rate equations.

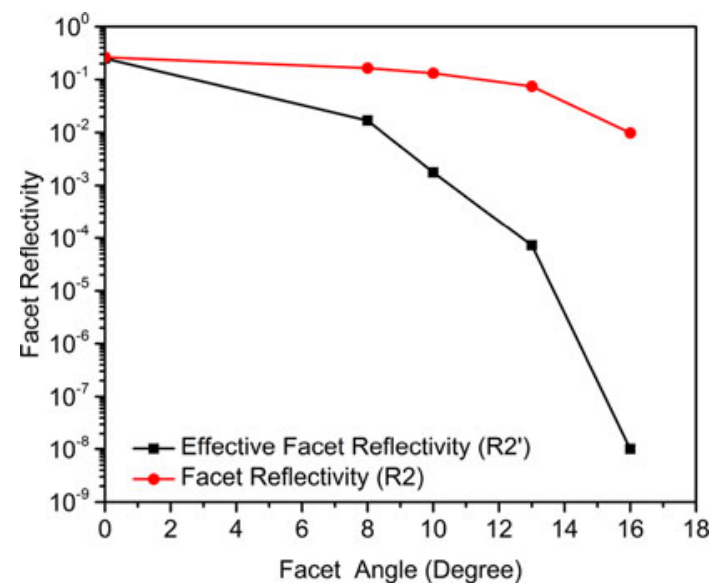

Fig. 11. The calculated facet reflectivity and the effective facet reflectivity as a function facet angle.

\section{CONCLUSION}

In summary, we have demonstrated high performance InAs/GaAs QDs lasers on offcut Si substrates with long lifetime by $\mathrm{MBE}$, and the first electrically pumped InAs/GaAs QD lasers monolithically grown on on-axis GaAs/Si (001) substrates by combined MOCVD and MBE techniques. In addition, we have reported the first studies of post-fabrication of various Si-based III-V QD light sources by means of FIB for use in silicon photonics. High performance RT c.w. lasing characteristics have been achieved from Si-based InAs/GaAs QD lasers with FIB-made front facet. Effectively reduced facet reflectivity has been achieved from angled facet devices, by focused $\mathrm{Ga}^{+}$ion 
beam milling of the front facet of the edge emitting Si-based InAs/GaAs QD laser, allowing the Si-based InAs/GaAs QD SLDs operating under c.w mode to be realized for the first time at room temperature. Our demonstration of the capability to post-fabricate various CMOS compatible electrically pumped c.w. Si-based light sources, including lasers and SLDs by a rapid and flexible FIB method opens up new opportunities toward monolithic integration of complex optoelectronics devices on the Si platform for silicon photonics.

\section{ACKNOWLEDGMENT}

The authors want to thank RENATECH program, CEA-Leti Minatec clean room staff and especially Y. Bogumilowcz, and Applied Materials for Fruitful discussion.

\section{REFERENCES}

[1] E. Tournie et al., "Metamorphic III-V semiconductor lasers grown on silicon," MRS Bulletin, vol. 41, pp. 218-223, 2016.

[2] R. E. Camacho-Aguilera et al., "An electrically pumped germanium laser," Opt. Express, vol. 20, pp. 11316-20, 2012.

[3] D. Liang and J. E. Bowers, "Recent progress in lasers on silicon," Nature Photon., vol. 4, pp. 511-517, 2010.

[4] A. Rickman, "The commercialization of silicon photonics," Nature Photon., vol. 8, pp. 579-582, 2014.

[5] D. Miller, "Device requirements for optical interconnects to silicon chips," Proc. IEEE, vol. 97, no. 7, pp. 1166-1185, Jul. 2010.

[6] G. T. Reed, G. Mashanovich, F. Gardes, and D. Thomson, "Silicon optical modulators," Nature Photon., vol. 4, pp. 518-526, 2010.

[7] M. Asghari and A. V. Krishnamoorthy, "Silicon photonics: Energyefficient communication," Nature Photon., vol. 5, pp. 268-270, 2011.

[8] L. Virot et al., "Germanium avalanche receiver for low power interconnects," Nature Commun., vol. 5, 2014, Art. no. 4957.

[9] A. D. Lee, Q. Jiang, M. C. Tang, Y. Y. Zhang, A. J. Seeds, and H. Y. $\mathrm{Liu}$, "InAs/GaAs quantum-dot lasers monolithically grown on $\mathrm{Si}, \mathrm{Ge}$, and Ge-on-Si substrates," IEEE J. Sel. Topics Quantum Electron., vol. 19, no. 4, pp. 1901107-1901107, Jul./Aug. 2013.

[10] H. H. Chang et al., "1310 nm silicon evanescent laser," Opt. Express, vol. 15, pp. 11466-11471, 2007.

[11] H. D. Kim, S. G. Kang, and C. H. Lee, "A low-cost WDM source with an ASE injected Fabry-Perot semiconductor laser," IEEE Photon. Technol. Lett., vol. 12, no. 8, pp. 1067-1069, Sep. 2000.

[12] M. Smit et al., "Generic foundry model for InP-based photonics," IET Optoelectron., vol. 5, pp. 187-194, 2011.

[13] Intel. Intel Silicon Photonics 100G PSM4 Optical Transceiver Brief, 2016. [Online]. Available: http://www.intel.com/content/www/us/en/ architecture-and-technology/silicon-photonics/optical-transceiver-100gpsm4-qsfp28-brief.html

[14] W. Burns, C. L. Chen, and R. Moeller, "Fiber-optic gyroscopes with broad-band sources," J. Lightw. Technol., vol. 1, no. 1, pp. 98-105, Mar. 1983.

[15] D. Huang et al., "Optical coherence tomography," Science, vol. 254, pp. $1178-1181,1991$.

[16] S. J. Park, C. H. Lee, K. T. Jeong, H. J. Park, J. G. Ahn, and K. H. Song, "Fiber-to-the-home services based on wavelength-divisionmultiplexing passive optical network," J. Lightw. Technol., vol. 22, no. 11, pp. 2582-2591, Nov. 2004.

[17] J. Lee, Y. Chung, and D. DiGiovanni, "Spectrum-sliced fiber amplifier light source for multichannel WDM applications," IEEE Photon. Technol. Lett., vol. 5, no. 12, pp. 1458-1461, Dec. 1993.

[18] L. Boivin and B. C. Collings, "Spectrum slicing of coherent sources in optical communications," Opt. Fiber Technol., vol. 7, pp. 1-20, 2001.

[19] Y. Wan etal., "Optically pumped $1.3 \mu \mathrm{m}$ room-temperature InAs quantumdot micro-disk lasers directly grown on (001) silicon," Opt. Lett., vol. 41 , pp. 1664-1667, 2016.

[20] Z. Wang et al., "Room-temperature InP distributed feedback laser array directly grown on silicon," Nature Photon., vol. 9, pp. 837-842, 2015.

[21] S. Wirths et al., "Lasing in direct-bandgap GeSn alloy grown on Si," Nature Photon., vol. 9, pp. 88-92, 2015.
[22] Y. H. Jhang et al., "Direct modulation of $1.3 \mu \mathrm{m}$ quantum dot lasers on silicon at $60^{\circ} \mathrm{C}, "$ Opt. Express, vol. 24, pp. 18428-18435, 2016.

[23] D. Liang, X. Huang, G. Kurczveil, M. Fiorentino, and R. Beausoleil, "Integrated finely tunable microring laser on silicon," Nature Photon., vol. 10, pp. 719-722, 2016.

[24] Y. L. Cao et al., "Hybrid III-V/silicon laser with laterally coupled Bragg grating," Opt. Express, vol. 23, pp. 8800-8808, 2015.

[25] G. Kurczveil, D. Liang, M. Fiorentino, and R. G. Beausoleil, "Robust hybrid quantum dot laser for integrated silicon photonics," Optics Express, vol. 24, pp. 16167-16174, 2016.

[26] E. Kleijn, M. K. Smit, and X. J. Leijtens, "Multimode interference reflectors: A new class of components for photonic integrated circuits," $J$. Lightw. Technol., vol. 31, no. 18, pp. 3055-3063, Sep. 2013.

[27] Z. Zhou, B. Yin, and J. Michel, "On-chip light sources for silicon photonics," Light: Sci. Appl., vol. 4, 2015, Art. no. e358.

[28] R. Fischer et al., "Growth and Properties of GaAs/AlGaAs on nonpolar substrates using molecular-beam epitaxy," J. Appl. Phys., vol. 58, pp. 374-381, 1985 .

[29] D. G. Deppe, K. Shavritranuruk, G. Ozgur, H. Chen, and S. Freisem, "Quantum dot laser diode with low threshold and low internal loss," Electron. Lett., vol. 45, pp. 54-55, 2009.

[30] H. Liu et al., "Optimizing the growth of $1.3 \mu \mathrm{m}$ InAs/InGaAs dots-in-awell structure," J. Appl. Phys., vol. 93, pp. 2931-2936, 2003.

[31] Z. T. Mi, J. Yang, P. Bhattacharya, G. X. Qin, and Z. Q. Ma, "Highperformance quantum dot lasers and integrated optoelectronics on $\mathrm{Si}$," Proc. IEEE, vol. 97, no. 7, pp. 1239-1249, Jul. 2009.

[32] S. F. Chichibu et al., "Origin of defect-insensitive emission probability in In-containing (Al, In, Ga)N alloy semiconductors," Nature Mater, vol. 5, pp. 810-816, 2006.

[33] Y. Arakawa and H. Sakaki, "Multidimensional quantum well laser and temperature dependence of its threshold current," Appl. Phys. Lett., vol. 40, pp. 939-941, 1982.

[34] R. Beanland et al., "Structural analysis of life tested $1.3 \mu \mathrm{m}$ quantum dot lasers," J. Appl. Phys., vol. 103, p. 014913, 2008.

[35] Y. Ebiko et al., "Island size scaling in InAs/GaAs self-assembled quantum dots," Phys. Rev. Lett., vol. 80, pp. 2650-2653, 1998.

[36] H. Liu et al., "Long-wavelength InAs/GaAs quantum-dot laser diode monolithically grown on Ge substrate," Nature Photon., vol. 5, pp. 416-419, 2011.

[37] A. Lee, Q. Jiang, M. Tang, A. Seeds, and H. Liu, "Continuous-wave InAs/GaAs quantum-dot laser diodes monolithically grown on $\mathrm{Si}$ substrate with low threshold current densities," Opt. Express, vol. 20, pp. 22181-22187, 2012.

[38] A. Y. Liu et al., "High performance continuous wave $1.3 \mu$ m quantum dot lasers on silicon," Appl. Phys. Lett., vol. 104, 2014, Art. no. 041104.

[39] T. Wang, H. Liu, A. Lee, F. Pozzi, and A. Seeds, "1.3- $\mu \mathrm{m}$ InAs/GaAs quantum-dot lasers monolithically grown on Si substrates," Opt. Express, vol. 19, pp. 11381-11386, 2011.

[40] M. Tang et al., "1.3- $\mu \mathrm{m}$ InAs/GaAs quantum-dot lasers monolithically grown on Si substrates using InAlAs/GaAs dislocation filter layers," Opt. Express, vol. 22, pp. 11528-11535, 2014.

[41] S. Chen et al., "1.3- $\mu \mathrm{m}$ InAs/GaAs quantum-dot laser monolithically grown on Si Substrates operating over $100{ }^{\circ} \mathrm{C}$, , Electron. Lett., vol. 50, pp. 1467-1468, 2014.

[42] Z. Mi, J. Yang, P. Bhattacharya, and D. Huffaker, "Self-organised quantum dots as dislocation filters: the case of GaAs-based lasers on silicon," Electron. Lett., vol. 42, p. 1, 2006.

[43] J. Wu, A. Lee, Q. Jiang, M. Tang, A. J. Seeds, and H. Liu, "Electrically pumped continuous-wave 1.3- $\mu \mathrm{m}$ InAs/GaAs quantum dot lasers monolithically grown on Si substrates," IET Optoelectron., vol. 8, pp. 20-24, 2014.

[44] A. Y. Liu, S. Srinivasan, J. Norman, A. C. Gossard, and J. E. Bowers, "Quantum dot lasers for silicon photonics [Invited]," Photon. Res., vol. 3, pp. B1-B9, 2015.

[45] J. Wu, S. M. Chen, A. Seeds, and H. Y. Liu, "Quantum dot optoelectronic devices: lasers, photodetectors and solar cells," J. Phys. D: Appl. Phys., vol. 48, 2015, Art. no. 363001.

[46] A. Y. Liu, R. W. Herrick, O. Ueda, P. M. Petroff, A. C. Gossard, and J. E. Bowers, "Reliability of InAs/GaAs quantum dot lasers epitaxially grown on silicon," IEEE J. Sel. Topics Quantum Electron., vol. 21, no. 6, pp. 690-697, Nov./Dec. 2015.

[47] S. M. Chen et al., "Electrically pumped continuous-wave III-V quantum dot lasers on silicon," Nature Photon., vol. 10, pp. 307-311, 2016.

[48] T. Wang et al., "The effect of growth temperature of GaAs nucleation layer on InAs/GaAs quantum dots monolithically grown on Ge substrates," Appl. Phys. Lett., vol. 100, 2012, Art. no. 052113. 
[49] M. Tang et al., "Optimizations of defect filter layers for 1.3- $\mu \mathrm{m}$ InAs/GaAs quantum-dot lasers monolithically grown on Si substrates," IEEE J. Sel. Topics Quantum Electron., vol. 22, no. 6, pp. 50-56, Nov./Dec. 2016.

[50] R. Alcotte et al., "Epitaxial growth of antiphase boundary free GaAs layer on $300 \mathrm{~mm} \mathrm{Si}(001)$ substrate by metalorganic chemical vapour deposition with high mobility," Appl. Mater., vol. 4, 2016, Art. no. 046101.

[51] K. Volz et al., "GaP-nucleation on exact $\mathrm{Si}(001)$ substrates for III/V device integration," J. Crystal Growth, vol. 315, pp. 37-47, 2011.

[52] A. Y. Liu et al., "Electrically pumped continuous-wave 1.3 mum quantumdot lasers epitaxially grown on on-axis (001) GaP/Si," Opt. Lett., vol. 42, pp. 338-341, 2017.

[53] S. M. Chen et al., "InAs/GaAs Quantum-Dot Superluminescent lightemitting diode monolithically grown on a Si substrate," ACS Photon., vol. 1, pp. 638-642, 2014.

[54] J. Schrauwen, D. Van Thourhout, and R. Baets, "Iodine enhanced focusedion-beam etching of silicon for photonic applications," J. Appl. Phys., vol. 102, 2007, Art. no. 103104.

[55] D. J. Moss et al., "Bragg gratings in silicon-on-insulator waveguides by focused ion beam milling," Appl. Phys. Lett., vol. 85, pp. 4860-4862, 2004.

[56] S. Chen et al., "Electrically pumped continuous-wave $1.3 \mu \mathrm{m}$ InAs/GaAs quantum dot lasers monolithically grown on on-axis $\mathrm{Si}$ (001) substrates," Opt. Express, vol. 25, pp. 4632-4639, 2017.

[57] J. R. Orchard et al., "In situ annealing enhancement of the optical properties and laser device performance of InAs quantum dots grown on $\mathrm{Si}$ substrates," Opt. Express, vol. 24, pp. 6196-6202, 2016.

[58] M. Tang et al., "Optimisation of the dislocation filter layers in $1.3-\mu \mathrm{m}$ InAs/GaAs quantum-dot lasers monolithically grown on $\mathrm{Si}$ substrates," IET Optoelectron., vol. 9, pp. 61-64, 2015.

[59] H. Liu et al., "Improved performance of $1.3 \mu \mathrm{m}$ multilayer InAs quantumdot lasers using a high-growth-temperature GaAs spacer layer," Appl. Phys. Lett., vol. 85, pp. 704-706, 2004

[60] J. Yang, P. Bhattacharya, and Z. Wu, "Monolithic integration of InGaAsGaAs quantum-dot laser and quantum-well electroabsorption modulator on silicon," IEEE Photon. Technol. Lett., vol. 19, no. 10, pp. 747-749, May 2007.

[61] M. Sugawara and M. Usami, "Quantum dot devices: Handling the heat," Nature Photon., vol. 3, pp. 30-31, 2009.

[62] Q. Jiang, M. Tang, S. Chen, J. Wu, A. Seeds, and H. Liu, "InAs/GaAs quantum-dot superluminescent diodes monolithically grown on a Ge substrate," Opt Express, vol. 22, pp. 23242-23248, 2014.

[63] L. H. Li, M. Rossetti, A. Fiore, L. Occhi, and C. Velez, "Wide emission spectrum from superluminescent diodes with chirped quantum dot multilayers," Electron. Lett., vol. 41, pp. 41-43, 2005.

[64] K. J. Zhou et al., "Quantum dot selective area intermixing for broadband light sources," Opt Express, vol. 20, pp. 26950-26957, 2012.

[65] S. M. Chen et al., "Hybrid quantum well/quantum dot structure for broad spectral bandwidth emitters," IEEE J. Sel. Topics Quantum Electron., vol. 19, no. 4, pp. 1900209-1900209, Jul./Aug. 2013.

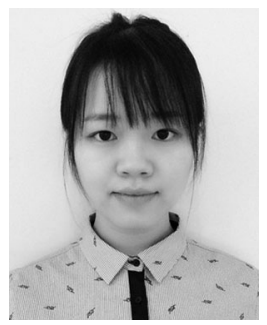

Mengya Liao received the B.Eng. (Hons.) degree in electronic and electrical engineering from University College London (UCL), London, U.K., in 2015, where she is currently working toward the Ph.D. degree at the Photonics group. Her research interests include optoelectronic device fabrication and molecular beam epitaxy of III-V compound semiconductors.

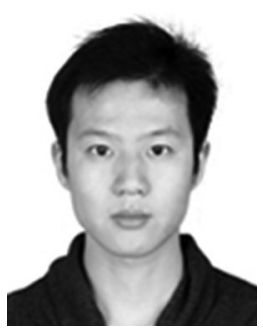

Siming Chen received the Ph.D. degree in electrical engineering from the University of Sheffield, Sheffield, U.K., in 2014. In 2013, he joined the Department of Electronic and Electrical Engineering, University College London, London, U.K., where he is now a Research Associate. He has been working on III-V and Si-based quantum dot optoelectronics devices, and silicon photonics.

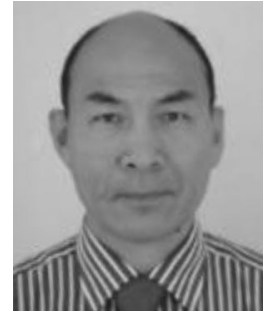

Suguo Huo received the Ph.D. degree in 1999 in materials science and engineering from the University of Sheffield, Sheffield, U.K. (1994-1997). From 1997 to 1998, he was a Research Associate in CRIST, Department of SECEE, University of Plymouth. From 1998 to 2000, he was a Postdoctal Research Fellow in Microstructues group, H.H. Wills Physics Laboratory, University of Bristol. From 2000 to 2009, he worked in Seagate Technology as a Senior Staff Engineer. Since 2009, he has been working in LCN, UCL as E/Ion Beam Manager. He received the Millennium year "Brounton Medal" from the University of Sheffield.



Si Chen was born in Henan, China, in 1992. He received the B.S. degree from the Huazhong University of Science and Technology, Wuhan, China, in 2013 and the M.S. degree in semiconductors from the University of Sheffield, Sheffield, U.K., in 2015, where he is currently working toward the Ph.D. degree. His research interests include semiconductor-based ultrafast optical switches.

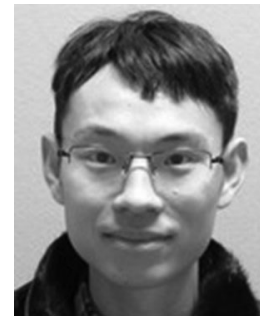

Jiang Wu obtained the B.S. degree from the University of Electronic Science and Technology of China, Sichuan Sheng, China. He obtained the M.Sc. and $\mathrm{Ph} . \mathrm{D}$. degrees in electrical engineering from the University of Arkansas, Fayetteville, AR, USA, in 2008 and 2011, respectively. In 2012, he joined the Photonics Group as a Research Associate on molecular beam epitaxy of III-V compound semiconductors and optoelectronic devices. Since 2015, he has been working as a Lecturer in the Department of Electronic and Electrical Engineering, University College London,

London, U.K.

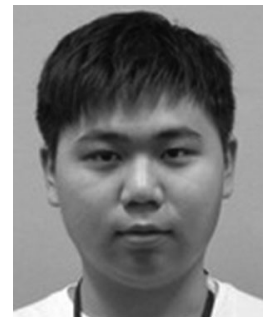

Mingchu Tang obtained the B.Eng. degree in electronic and electrical engineering from the University of Sussex, Brighton, U.K., in 2011 and the M.Sc. degree in nanotechnology from the University College London, London, U.K., in 2012. Since 2012, he has been working toward the Ph.D. degree on molecular beam epitaxy of III-V compound semiconductors and optoelectronic devices at the Photonics group His research interests include III-V quantum dot optoelectronic devices monolithically grown on group III-V and IV platform.

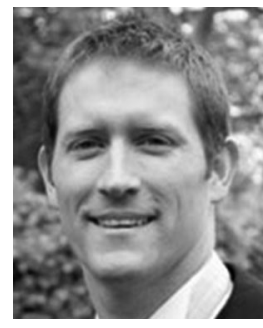

Ken Kennedy received the B.Sc. degree in optoelectronics from Heriot-Watt University, Edinburgh, U.K., in 1999, and the Ph.D. degree in electronic and electrical engineering from the University of Sheffield, Sheffield, U.K., in 2009. He immediately joined Hewlett Packard, Ipswich, UK, as a Manufacturing/R\&D Engineer working on telecom wavelength emitters and detectors. In 2004, he joined Agilent Technologies Ltd., Singapore as a Technology Transfer Engineer. He currently works in the EPSRC National Centre, University of Sheffield for III-V technologies and is responsible for the device fabrication of a variety of devices and materials. His research interests include semiconductor laser and emitter technologies. 


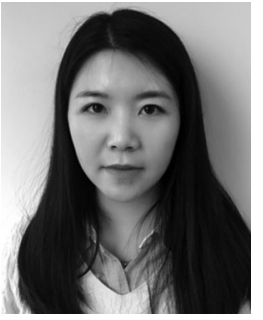

Wei $\mathrm{Li}$ received the M.Sc. degree in electronic engineering from the University of Sheffield, Sheffield, U.K., in 2012, where she is currently working toward the Ph.D. degree in electronic and electrical engineering. Her research has been concerned with transmission electron microscopy and optical characterization of InGaAs/GaAs on Si quantum dot structures.

Saurabh Kumar photograph and biography not available at the time of publication.

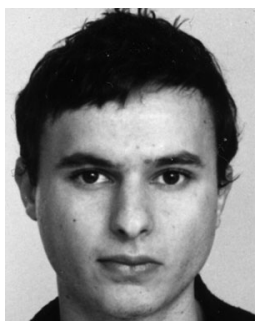

Mickael Martin received the B.Sc. degree in physics and the M.S. degree in materials engineering from Strasbourg University, Strasbourg, France, in 2001 and 2003, respectively. He joined the Microelectronics Technology Laboratory (CNRS-LTM), University Grenoble Alpes, Grenoble, France, in 2005. He is currently serving as a Process Engineer in the nanomaterials team, working on the III-V-on-silicon MOCVD epitaxy.



Thierry Baron received the Ph.D. degree from University Grenoble Alpes, Grenoble, France, in 1996. $\mathrm{He}$ is the Director of research at Centre Nationnal de la Recherche Scientifique. He is currently the Head of the Laboratoire des Technologies de la Microelectronique located on the CEA-LETI MINATEC's site in Grenoble. He is the Co-executive Director of an advanced technological platform. He is involved in the field of nanomaterials elaboration and their integration in devices. He is currently studying the growth of III-V semiconductors by MOCVD and their monolithic integration on $\mathrm{Si} \mathrm{CMOS}$ platform for optoelectronic and nanoelectronic devices.



Chaoyuan Jin received the Ph.D. degree from the University of Sheffield, Sheffield, U.K., in 2008 From 2008 to 2010, he was awarded a JSPS Postdoctoral Fellowship and worked on quantum dot switches at Kobe University, Japan. In 2010, he joined the Eindhoven University of Technology, the Netherlands, as a Postdoctoral Researcher, working on ultrafast control of cavity quantum electrodynamics based on single quantum dots in photonic crystal cavities. From 2013 to 2015, he joined EFFECT Photonics B.V. In his role as a Senior Photonics Scientist, he developed high-speed optical transceivers for short-reach optical communications based on generic photonic integration technologies. He is currently working as a Vice-Chancellor's Fellow, developing research programs on ultrafast photonic devices in the Department of Electronic and Electrical Engineering, University of Sheffield, UK. His research activities focus on nanophotonic devices for optical signal and quantum information processing, including optical switches, lasers, single-photon sources, and nanophotonic integrated circuits.

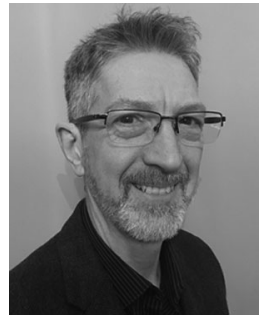

Ian Ross received the Ph.D. degree from the University of Liverpool, Liverpool, U.K. in collaboration with Unilever, London, U.K., in 1998. He is currently a Senior Experimental Officer in the Department of Electronic and Electrical Engineering, University of Sheffield, U.K. He worked extensively in the field of advanced materials characterization during his $\mathrm{Ph} . \mathrm{D}$. His research interests include the application of aberration corrected electron microscopy to semiconductor and functional nanostructures.

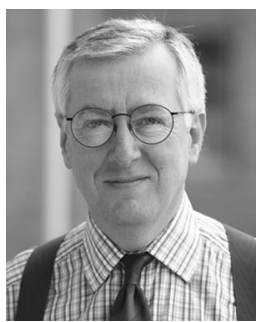

Alwyn Seeds received the B.Sc., Ph.D., and D.Sc. degrees from the University of London, London, U.K From 1980 to 1983, he was a Staff Member at Lincoln Laboratory, Massachusetts Institute of Technology, where he worked on GaAs monolithic millimeterwave integrated circuits for use in phased-array radar. Following three years as a Lecturer in telecommunications at Queen Mary College, University of London, he moved to University College London in 1986 , where he is now a Professor of opto-electronics and the Head of the Department of Electronic and Electrical Engineering. He has published more than 350 papers on microwave and optoelectronic devices and their systems applications. His current research interests include semiconductor optoelectronic devices, wireless and optical communication systems.

Dr. Seeds has been a Member of the Board of Governors and the VicePresident for Technical Affairs of the IEEE Photonics Society. He has served on the programme committees for many international conferences. He is the Co-founder of Zinwave, a manufacturer of wireless over fibre systems. He was awarded the Gabor Medal and Prize of the Institute of Physics in 2012. He is a Fellow of the Royal Academy of Engineering.

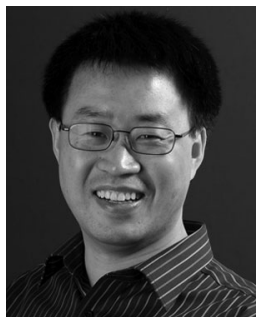

Huiyun Liu received the Ph.D. degree in semiconductor science from the Institute of Semiconductors, Chinese Academy of Sciences, Beijing, China. In August 2001, he joined the EPSRC National Centre for III-V Technologies, University of Sheffield. He was responsible for the development of molecular beam epitaxy growth of semiconductor materials for the U.K. academic and industrial research community. In 2007, he was awarded Royal Society University Research Fellow and started his academic career in the Department of Electronic and Electrical Engineering, University College London, where he is currently a Professor of semiconductor photonics. His research interests include the nanometer-scale engineering of low-dimensional semiconductor structures (such as quantum dots, quantum wires, and quantum wells) by using molecular beam epitaxy and the development of novel optoelectronic devices including lasers, detectors, and modulators by developing novel device process techniques. He has co-authored more than 300 papers in the area of semiconductor materials and devices. 